ApJSup accepted (Spitzer Special Issue)

Preprint typeset using $\mathrm{LAT}_{\mathrm{E}} \mathrm{X}$ style emulateapj v. 6/22/04

\title{
OBSERVATIONS OF ULTRALUMINOUS INFRARED GALAXIES WITH THE INFRARED SPECTROGRAPH ON THE SPITZER SPACE TELESCOPE: EARLY RESULTS ON MRK 1014, MRK 463, AND UGC 5101 ${ }^{1}$
}

L. Armus ${ }^{2}$, V. Charmandaris ${ }^{3,6}$, H.W.W. Spoon ${ }^{3}$, J.R. Houck ${ }^{3}$, B.T. Soifer ${ }^{2}$, B.R. Brandi ${ }^{4}$, P.N. Appleton ${ }^{2}$, H.I. Teplitz ${ }^{2}$, S.J.U. Higdon ${ }^{3}$, D.W. Weedman ${ }^{3}$, D. Devost ${ }^{3}$, P.W. Morris ${ }^{2}$, K.I. UChida ${ }^{3}$, J. VAn Cleve ${ }^{5}$, D.J. Barry ${ }^{3}$, G.C. Sloan ${ }^{3}$, C.J. Grillmair ${ }^{2}$, M.J. Burgdorf ${ }^{2}$, S.B. Fajardo-Acosta ${ }^{2}$, J.G. Ingalls ${ }^{2}$, J. Higdon $^{3}$ L. Hao $^{3}$, J. $^{2}$

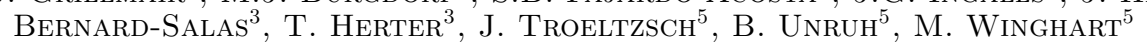

ApJSup accepted (Spitzer Special Issue)

\begin{abstract}
We present spectra taken with the Infrared Spectrograph ${ }^{7}$ on Spitzer covering the $5-38 \mu \mathrm{m}$ region of three Ultraluminous Infrared Galaxies (ULIRGs): Mrk $1014(z=0.163)$, and Mrk $463(z=0.051$ ), and UGC $5101(z=0.039)$. The continua of UGC 5101 and Mrk 463 show strong silicate absorption suggesting significant optical depths to the nuclei at $10 \mu \mathrm{m}$. UGC 5101 also shows the clear presence of water ice in absorption. PAH emission features are seen in both Mrk 1014 and UGC 5101, including the $16.4 \mu \mathrm{m}$ line in UGC 5101. The fine structure lines are consistent with dominant AGN power sources in both Mrk 1014 and Mrk 463. In UGC 5101 we detect the [NeV] $14.3 \mu \mathrm{m}$ emission line providing the first direct evidence for a buried AGN in the mid-infrared. The detection of the $9.66 \mu \mathrm{m}$ and $17.03 \mu \mathrm{m} \mathrm{H}_{2}$ emission lines in both UGC 5101 and Mrk 463 suggest that the warm molecular gas accounts for $22 \%$ and $48 \%$ of the total molecular gas masses in these galaxies.

Subject headings:
\end{abstract}

\section{INTRODUCTION}

Ultraluminous Infrared Galaxies (ULIRGs), i.e. those galaxies with infrared luminosity $\mathrm{L}_{I R} \gtrsim 10^{12} \mathrm{~L}_{\odot}$, have the power output of quasars yet emit nearly all of their energy in the mid and far-infrared part of the spectrum. Most ULIRGs are found in interacting and merging systems (e.g. Armus, Heckman \& Miley 1987; Sanders, et al. 1988a; Murphy, et al. 1996), where the merger has driven gas and dust towards the remnant nucleus, fueling a massive starburst, and either creating or fueling a nascent AGN (Mihos \& Hernquist 1996). ULIRGs are rare in the local Universe, comprising only $3 \%$ of the IRAS Bright Galaxy Survey (Soifer et al. 1987), yet at $z>2-3$, ULIRGs may account for the bulk of all starformation activity and dominate the far-infrared background (e.g. Blain, et al. 2002).

Observations with the ISO satellite greatly expanded our understanding of the mid-infrared spectra of ULIRGs (e.g., Genzel et al. 1998; Lutz et al. 1999; Rigopoulou et al. 1999; Sturm et al. 2002; Tran et al. 2001). Diagnostic diagrams based on fine structure and aromatic emission features (UIB's, or PAH's) allowed some ULIRGs to be classified according to their dominant ionization mechanism. However, the complexities of the ULIRG spectra, the fact that many are likely composite AGN

\footnotetext{
1 based on observations obtained with the Spitzer Space Telescope, which is operated by the Jet Propulsion Laboratory, California Institute of Technology, under NASA contract 1407

2 Spitzer Science Center, MS 220-6, Caltech, Pasadena, CA 91125

3 Astronomy Department, Cornell University, Ithaca, NY 14853

${ }^{4}$ Leiden University, 2300 RA Leiden, The Netherlands

5 Ball Aerospace \& Technologies Corp., 1600 Commerce St., Boulder CO, 80301

6 Chercheur Associé, Observatoire de Paris, F-75014, Paris, France

7 The IRS was a collaborative venture between Cornell University and Ball Aerospace Corporation funded by NASA through the Jet Propulsion Laboratory and the Ames Research Center
}

and starburst sources, and the limitations in sensitivity of the ISO spectrometers, left many ULIRGs, even at relatively low redshift, beyond the reach of these methods until now.

In order to adequately sample the local ULIRG population, we are obtaining mid-infrared spectra of a large number $(>100$ ) of ULIRGs having $0.02<z<0.93$ with the Infrared Spectrograph (IRS) on Spitzer, as part of the IRS guaranteed time program. These sources are chosen from the complete Bright Galaxy Sample (Soifer et al. 1987), the 1-Jy (Kim \& Sanders 1998) and 2-Jy (Strauss et al. 1992) samples, and the FIRST/IRAS radio-farIR sample of Stanford et al. (2000). In this letter, we present the first results from this program, focusing on three nearby ULIRGs (UGC 5101, Mrk 463, and Mrk 1014) whose spectra reflect the range of properties we expect from the sample as a whole.

UGC $5101(z=0.039)$ has a single, very red nucleus within a disturbed morphology suggestive of a recent interaction and merger. Optically, UGC 5101 is classified as a LINER (Veilleux et al. 1995). It has a high brightness temperature $\left(\mathrm{T}>10^{7} \mathrm{~K}\right)$ radio nucleus at $1.6 \mathrm{GHz}$ which is resolved with the VLBA (Lonsdale et al. 1995). ISO SWS and PHT-S spectroscopy (Genzel el al. 1998) indicate a powerful, circumnuclear starburst. Ground-based, high-resolution mid-IR imaging (Soifer et al. 2000) indicates that $\approx 60 \%$ of the IRAS flux at $12 \mu \mathrm{m}$ comes from the central four arcsec, and that nearly half of this arises in an unresolved core. Based upon its IRAS colors, UGC 5101 is classified as a cold, starburst-dominated, far-infrared source. However, XMM data indicate an obscured, but luminous, hard $\mathrm{x}-$ ray source with $\mathrm{L}_{x}(2-10 \mathrm{keV}) \sim 5 \times 10^{42} \mathrm{erg} \mathrm{s}^{-1}$ and $\mathrm{L}_{x}(2-10 \mathrm{keV}) / \mathrm{L}_{I R} \sim 0.002$ suggestive of a buried AGN (Imanishi, et al. 2003). Here, we present the first direct infrared evidence for an AGN in UGC 5101.

$\operatorname{Mrk} 463(z=0.0508)$ is a merging system with two nuclei separated by about four arcseconds (Mazzarella, 
TABLE 1

OBSERVATION LoG

\begin{tabular}{cccc}
\hline \hline & UGC 5101 & Mrk 1014 & Mrk 463e \\
\hline date & 15 Nov 2003 & 7 Jan 2004 & 7 Jan 2004 \\
& (23 Mar 2004) & & \\
PU target & BD+62 1078 & HD121829 & HD12382 \\
SL1 & $6 \times 14 \mathrm{sec}$ & $6 \times 14 \mathrm{sec}$ & $6 \times 14 \mathrm{sec}$ \\
SL2 & $6 \times 14 \mathrm{sec}$ & $6 \times 14 \mathrm{sec}$ & $6 \times 14 \mathrm{sec}$ \\
LL1 & $4 \times 30 \mathrm{sec}$ & $4 \times 30 \mathrm{sec}$ & $6 \times 14 \mathrm{sec}$ \\
LL2 & $4 \times 30 \mathrm{sec}$ & $4 \times 30 \mathrm{sec}$ & $6 \times 14 \mathrm{sec}$ \\
SH & $2 \times 30 \mathrm{sec}$ & $12 \times 30 \mathrm{sec}$ & $12 \times 30 \mathrm{sec}$ \\
LH & $2 \times 60 \mathrm{sec}$ & $8 \times 60 \mathrm{sec}$ & $8 \times 60 \mathrm{sec}$ \\
& & & \\
\hline
\end{tabular}

Note. - IRS observation details, including dates, peakup target, and integration times, are given for each galaxy. For each slit we list the cycles and ramp durations, where $6 \mathrm{x} 14 \mathrm{sec}$ indicates six cycles of $14 \mathrm{sec}$ ramps, including both nod positions. The SL and LL observations for UGC 5101 were performed on 23 March 2004, while the $\mathrm{SH}$ and $\mathrm{LH}$ observations were performed on 15 November 2003.

et al. 1991). Both nuclei have Seyfert 2 optical spectra (Shuder \& Osterbrock 1981), but broad lines are seen in scattered optical (Miller \& Goodrich 1990) and direct near-infrared light (Goodrich, Veilleux \& Hill 1994; Veilleux et al. 1997). The eastern nucleus (Mrk463e) is much redder $(V-K=6.8 \mathrm{mag})$, and has a luminous, steep-spectrum radio core (Mazzarella et al.). Although the far-infrared luminosity of Mrk $463\left(5 \times 10^{11} \mathrm{~L}_{\odot}\right)$ is slightly less than the canonical ULIRG cutoff of $10^{12} \mathrm{~L}_{\odot}$, the bolometric luminosity of this system is very high, and we refer to it as a ULIRG for the remainder of this paper.

Mrk $1014(z=0.1631)$ is a radio-quiet, infrared luminous QSO with broad optical emission lines (FWHM $\mathrm{H} \beta>4000 \mathrm{~km} \mathrm{~s}^{-1}$ ) and twin tidal tails indicative of a recent interaction and merger (MacKenty \& Stockton 1984). Both Mrk 463 and Mrk 1014 are warm, farinfared sources with $\mathrm{S}_{25} / \mathrm{S}_{60}=0.74$ and 0.27 , respectively (Sanders, et al. 1988b).

Throughout the paper, we will adopt a flat, $\Lambda$ dominated Universe $\left(H_{0}=70 \mathrm{~km} \mathrm{~s}^{-1} \mathrm{Mpc}^{-1}, \Omega_{M}=0.3\right.$, $\left.\Omega_{\Lambda}=0.7\right)$. The luminosity distances to UGC 5101, Mrk 463, and Mrk 1014 are then $170 \mathrm{Mpc}, 223 \mathrm{Mpc}$, and 774 $\mathrm{Mpc}$, and one arcsec subtends $0.76,0.98$, and $2.77 \mathrm{kpc}$ in projection, respectively.

\section{OBSERVATIONS}

Details of the observations are given in Table 1 . The IRS is fully described in Houck et al. (2004). All three ULIRGs were observed in the two low-resolution $(64<R<128$; Short-Low and Long-Low or SL \& LL) and two high-resolution $(R \sim 600$; Short-High and LongHigh or SH \& LH) IRS modules, using the Staring Mode Astronomical Observing Template (AOT). High accuracy blue peak-ups were performed on nearby 2MASS stars before offsetting to the target galaxies. For Mrk463, the eastern nucleus, Mrk463e, was centered in the IRS slits in all cases. While the separation of the nuclei is comparable to or less than the widths of the IRS slits, we expect Mrk463e to dominate at all IRS wavelengths.

\section{DATA REDUCTION AND ANALYSIS}

All spectra were reduced using the IRS pipeline at the Spitzer Science Center. This reduction includes ramp

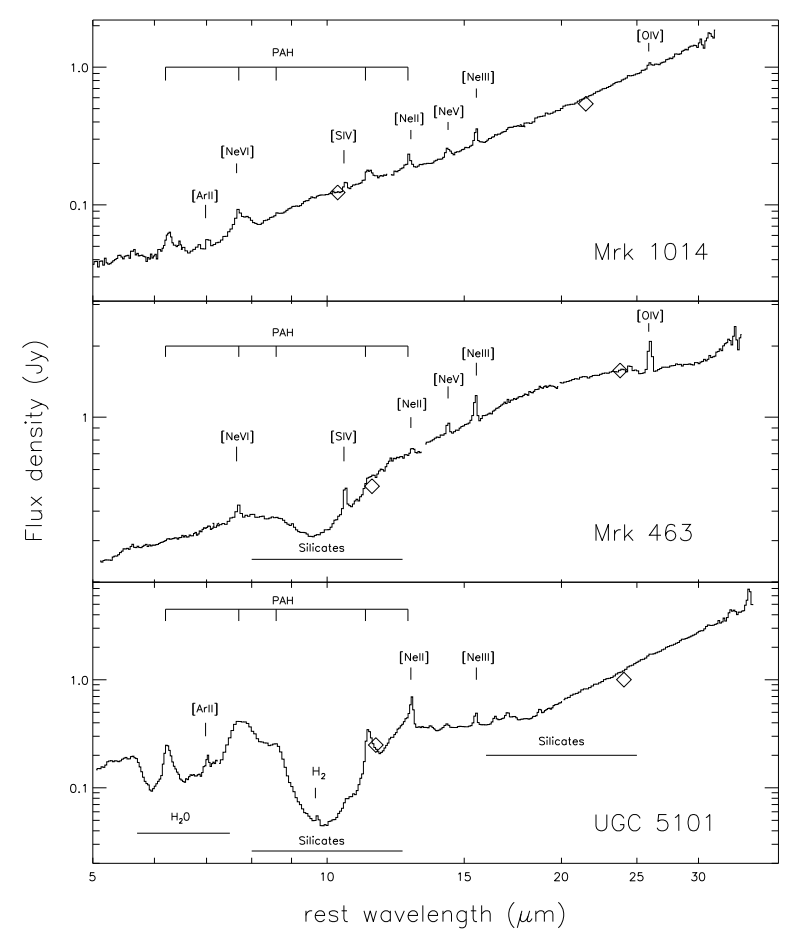

FIG. 1.- IRS Short-Low \& Long-Low spectra of Mrk 1014, Mrk 463e and UGC 5101. Prominent emission lines and absorption bands (the latter indicated by horizontal bars) are marked. Open diamonds are the IRAS $12 \mu \mathrm{m}$ and $25 \mu \mathrm{m}$ points.

fitting, dark sky subtraction, droop correction, linearity correction, flat fielding, and wavelength and flux calibration (see Chapter 7 of the Spitzer Observers Manual and Decin et al. 2004 for details). The SL and LL data have had local background light subtracted, by differencing the two nod positions along the slit, before spectral extraction. As a final step, we have normalized the SL and LL 1D spectra upwards to match the 12 and $25 \mu \mathrm{m}$ IRAS FSC data (Moshir et al. 1990). The final, average SL and LL spectra are displayed in Fig.1.

Since the SH and LH slits are too small for on-slit background subtraction, we have subtracted the expected background flux through each slit based on the model of Reach, et al. (2004). The SH and LH spectra were then scaled, on an order by order basis, to the corresponding low-resolution data. The average $\mathrm{SH}$ and $\mathrm{LH}$ spectra are shown in Figs 2 \& 3, respectively. While most high-res orders line up very well, there are slight offsets and residual curvature still visible in some orders (e.g. the bluest $\mathrm{SH}$ orders of Mrk 1014 and at rest wavelengths of $12 \mu \mathrm{m}$ and $22 \mu \mathrm{m})$. Noisy areas at the red end of the $\mathrm{SH}$ and LH orders in order overlap regions are not shown or used in the fitting process. In $\mathrm{SH}$, these noisy areas amount to typically 10-30 pixels at the red end of orders 13-20, corresponding to an area of decreased responsivity on the array.

\section{RESULTS}

\subsection{Continuum \& Absorption Features}

The continua of Mrk 1014, Mrk 463e and UGC 5101 are strikingly different. The low-resolution spectrum of UGC 5101 is a blend of a water ice (most easily seen as a strong absorption under the $6.2 \mu \mathrm{m}$ PAH emission fea- 


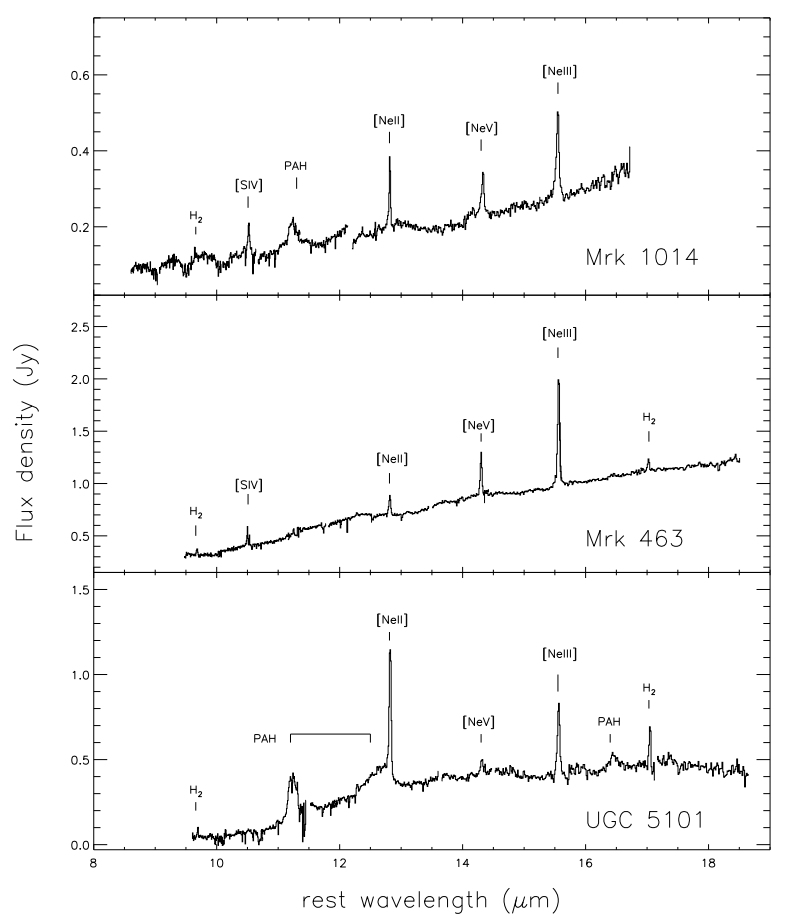

Fig. 2.- IRS Short-High spectra of Mrk 1014, Mrk 463e and UGC 5101. Prominent emission lines are marked.

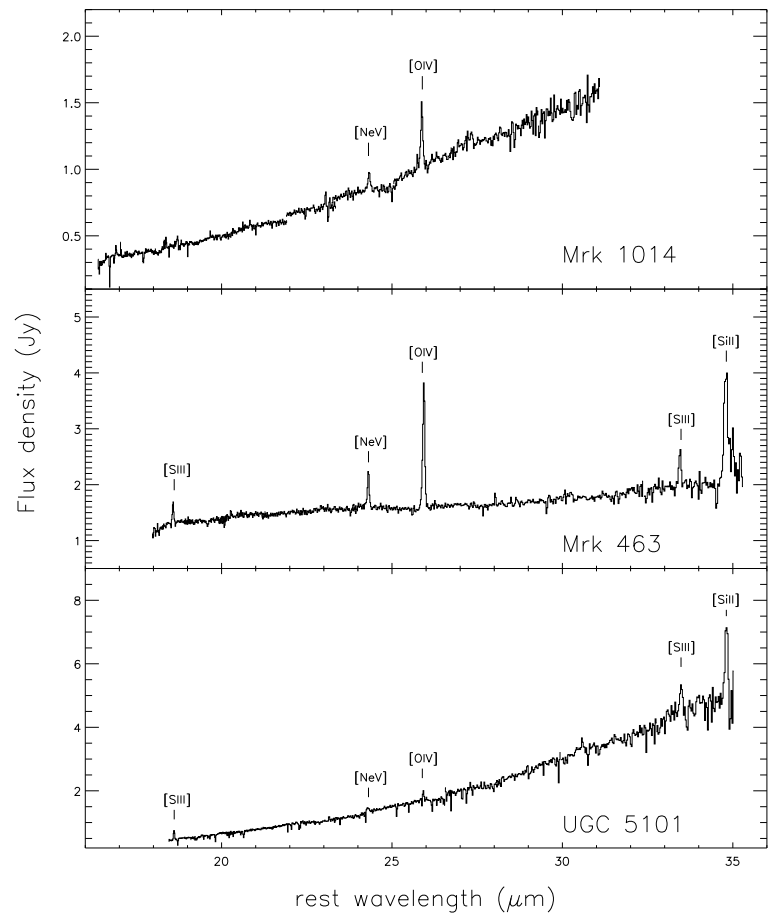

FIG. 3.- IRS Long-High spectra of Mrk 1014, Mrk 463e and UGC 5101. Prominent emission lines are marked.

ture), and silicate-absorbed (at $9.7 \mu \mathrm{m}$ and $18 \mu \mathrm{m}$ ) starburst spectrum (see Fig. 1). Much of the silicate absorption is filled in by the flanking PAH emission features. Adopting a smooth continuum anchored at $5.3-5.6 \mu$, $14 \mu \mathrm{m}$ and $34 \mu \mathrm{m}$, we derive optical depths of $\tau_{6}=0.75$, $\tau_{9.7} \geq 2$, and $\tau_{18} \geq 0.4$. The extinction to the central source is thus at least $A_{V}=15-35$ mag. As suggested by Spoon et al. (2002), the water ice features may indicate the presence of shielded molecular clouds along the line of sight to the nucleus. While the $5.7-7.5 \mu \mathrm{m}$ absorption is dominated by water ice, the structure visible from $6.8-7.5 \mu \mathrm{m}$ is consistent with hydrocarbon absorption at the level $\left(\tau_{6.85} \sim 0.16\right)$ expected from the $3.4 \mu \mathrm{m}$ band (Imanishi et al. 2001), assuming an optical depth ratio of $\tau_{6.85} / \tau_{3.4}=0.238$ (as measured for SgrA* by Chiar et al. 2000).

Mrk 1014 shows no obvious silicate absorption, suggesting a rather clean line of sight at $10 \mu \mathrm{m}$ to the Seyfert 1 nucleus. Mrk 463e is intermediate in its continuum properties between UGC 5101 and Mrk 1014. The most obvious continuum feature is the silicate absorption at $9.7 \mu \mathrm{m}$. Adopting a power-law continuum from $7-14 \mu \mathrm{m}$, we derive an optical depth $\tau_{9.7}=0.46$, corresponding to an $\mathrm{A}_{V}=3-8$ mag.

\subsection{Emission Features}

At high resolution, the IRS spectra of Mrk 1014, Mrk 463e and UGC 5101 are dominated by unresolved atomic, fine-structure lines of $\mathrm{Ne}, \mathrm{O}, \mathrm{Si}$, and $\mathrm{S}$, covering a large range in ionization potential (see Figs. $2 \& 3$ and Table 2 ). Ratios of these lines can be used to gauge the dominant ionizing source - whether it be hot stars or an active nucleus. Some features, e.g. the $[\mathrm{NeV}]$ lines at 14.3 and $24.3 \mu \mathrm{m}$, imply the presence of an AGN by their very detection in an integrated spectrum of a galaxy, since it takes $97.1 \mathrm{eV}$ to ionize [NeIV], and this is too large to be produced by OB stars. The same is not true for [OIV], since it takes only $55 \mathrm{eV}$ to ionize [OIII], and in fact the $25.9 \mu \mathrm{m}$ line has been seen in a number of starburst galaxies (e.g., Lutz et al. 1998).

The $[\mathrm{NeV}] 14.3 /[\mathrm{NeII}] 12.8$ and $[\mathrm{OIV}] 25.9 /[\mathrm{NeII}] 12.8$ line flux ratios in Mrk 1014 and Mrk 463e suggest that nearly all the ionizing flux in these sources $(>80-$ $90 \%$ based upon the simple scaling models in Sturm et al. 2002) comes from the active nuclei. In addition, the $[\mathrm{NeII}] 12.8 /[\mathrm{NeIII}] 15.6,[\mathrm{NeV}] 14.3 /[\mathrm{NeIII}] 15.6$, and $[\mathrm{NeV}] 14.3 /[\mathrm{NeV}] 24.3$ line flux ratios suggest that the electron densities are low $\left(10^{2}-10^{3} \mathrm{~cm}^{-3}\right)$ and that the ionization parameters and spectral indices are in the ranges of $-1<\log \mathrm{U}<-2$ and $\alpha \leq-1$ for both sources (Voit 1992, Sturm et al. 2002). Weak [NeVI] $7.65 \mu \mathrm{m}$ emission is also present in Mrk 1014 and Mrk 463e (see Fig.1), but it is blended with the $7.7 \mu \mathrm{m}$ PAH feature and possibly even Pf- $\alpha$. A proper separation of these components can only be done with IRS high-res data for galaxies with $z \geq 0.33$.

In UGC 5101 we have clearly detected a $[\mathrm{NeV}] 14.3 \mu \mathrm{m}$ line with a flux of $5.2( \pm 0.7) \times 10^{-21} \mathrm{~W} \mathrm{~cm}^{-2}$ indicating a buried AGN. The measured [NeV]14.3 flux is approximately a factor of three below the upper limit set by Genzel et al. (1998). We also detect the [OIV] $25.89 \mu \mathrm{m}$ line with a flux of $5.5( \pm 1.4) \times 10^{-21} \mathrm{~W} \mathrm{~cm} \mathrm{~cm}^{-2}$. A faint $[\mathrm{NeV}] 24.3 \mu \mathrm{m}$ line is also visible in the LH spectrum. The [SIII] $18.7 /[\mathrm{SIII}] 33.4$ and the $[\mathrm{NeV}] 14.3 /[\mathrm{NeV}] 24.3$ line flux ratios imply electron densities at or below $10^{2} \mathrm{~cm}^{-3}$, and the [NeIII $] 15.5 /[\mathrm{NeII}] 12.8$ line flux ratio implies a moderate excitation starburst (Verma et al. 2003). While the $[\mathrm{NeV}] 14.3 /[\mathrm{NeII}] 12.8$ and the $[\mathrm{OIV}] 25.9 /[\mathrm{NeII}] 12.8$ line flux ratios $(\sim 0.1$ in each case $)$ are consistent with an AGN contribution of $<10 \%$ to 
TABLE 2

EMISSION FEATURES

\begin{tabular}{|c|c|c|c|}
\hline Line $\mu \mathrm{m}$ & UGC 5101 & Mrk 1014 & Mrk 463e \\
\hline PAH 6.2 & $190(11)$ & $14.4(1.8)$ & \\
\hline & 0.258 & 0.048 & \\
\hline$[A r I I] 6.98$ & $\begin{array}{c}15.2(2.5)^{a} \\
0.018\end{array}$ & & \\
\hline$[\mathrm{NeVI}] 7.65$ & & $\begin{array}{c}5.5(0.5)^{b} \\
0.022\end{array}$ & $\begin{array}{c}18.7(2.1)^{b} \\
0.009\end{array}$ \\
\hline PAH 7.7 & $\begin{array}{c}560(26) \\
0.575\end{array}$ & $\begin{array}{c}32(5)^{b} \\
0.135\end{array}$ & $\begin{array}{c}33(6)^{b} \\
0.029\end{array}$ \\
\hline $\mathrm{H}_{2} 9.66$ & $\begin{array}{c}3.7(0.7) \\
0.034\end{array}$ & $\begin{array}{c}1.6(0.5) \\
0.007\end{array}$ & $\begin{array}{c}4.8(0.8) \\
0.003\end{array}$ \\
\hline$[S I V] 10.51$ & $\leq 2.4$ & $\begin{array}{c}7.9(0.9) \\
0.033\end{array}$ & $\begin{array}{c}9.0(1.2) \\
0.010\end{array}$ \\
\hline PAH 11.3 & $\begin{array}{c}106(10) \\
0.284\end{array}$ & $\begin{array}{c}4.6(0.8) \\
0.017\end{array}$ & \\
\hline$[N e I I] 12.81$ & $\begin{array}{c}55.2(2.5) \\
0.080\end{array}$ & $\begin{array}{c}7.8(0.8) \\
0.031\end{array}$ & $\begin{array}{c}11.6(0.7) \\
0.010\end{array}$ \\
\hline$[\mathrm{NeV}] 14.3$ & $\begin{array}{c}5.2(0.7) \\
0.009\end{array}$ & $\begin{array}{c}6.8(0.6) \\
0.029\end{array}$ & $\begin{array}{c}18.3(0.8) \\
0.015\end{array}$ \\
\hline$[N e I I I] 15.55$ & $\begin{array}{c}23.9(1.4) \\
0.050\end{array}$ & $\begin{array}{c}13.3(1.1) \\
0.054\end{array}$ & $\begin{array}{c}51.8(2.5) \\
0.052\end{array}$ \\
\hline PAH 16.4 & $\begin{array}{c}14.5(1.9) \\
0.031\end{array}$ & & \\
\hline $\mathrm{H}_{2} 17.03$ & $\begin{array}{c}7.2(1.4) \\
0.015\end{array}$ & & $\begin{array}{c}3.8(0.5) \\
0.004\end{array}$ \\
\hline$[S I I I] 18.71$ & $\begin{array}{c}9.8(0.7) \\
0.025\end{array}$ & & $\begin{array}{c}15.0(1.6) \\
0.013\end{array}$ \\
\hline$[\mathrm{NeV}] 24.31$ & $\begin{array}{c}4.9(1.0) \\
0.009\end{array}$ & $\begin{array}{c}5.1(0.5) \\
0.017\end{array}$ & $\begin{array}{c}20.4(1.7) \\
0.030\end{array}$ \\
\hline$[O I V] 25.89$ & $\begin{array}{c}5.5(1.4) \\
0.010\end{array}$ & $\begin{array}{c}13.5(1.5) \\
0.040\end{array}$ & $\begin{array}{c}72.3(1.2) \\
0.117\end{array}$ \\
\hline$[S I I I] 33.48$ & $\begin{array}{c}13.0(2.5) \\
0.016\end{array}$ & & $\begin{array}{c}13.5(1.6) \\
0.033\end{array}$ \\
\hline$[S i I I] 34.81$ & $\begin{array}{l}36(6) \\
0.047\end{array}$ & & $\begin{array}{c}30.3(5.9) \\
0.076\end{array}$ \\
\hline
\end{tabular}

Note. - For each line we give the central wavelength in microns, the flux, in units of $10^{-21} \mathrm{Wcm}^{-2}$ and the equivalent width, in microns, directly below. Formal uncertainties in the line fits (all single Gaussians except where noted) are listed in parentheses next to the fluxes. However, true uncertainties in the absolute line fluxes are generally $\sim 20-25 \%$. All lines were measured using the SMART spectral reduction package (Higdon et al. 2004). ${ }^{a}$ The [ArII]6.98 line is blended with the $\mathrm{H}_{2} \mathrm{~S}(5)$ line in UGC 5101. ${ }^{b}$ The $[\mathrm{NeVI}] 7.65$ line is blended with the $7.7 \mu \mathrm{m}$ PAH feature in Mrk 1014 and Mrk 463e, and a two-component Gaussian has been used to separate the unresolved and broad components.

the total luminosity in this source (Sturm et al. 2002), the large optical depth to the nucleus, as evidenced by the deep silicate absorption, leaves open the possibility that the true contribution of the AGN to the bolometric power output in UGC 5101 may be larger than revealed by the mid-IR emission lines.

The $\mathrm{H}_{2} \mathrm{~S}(3) 9.66 \mu \mathrm{m}$ and $\mathrm{S}(1) 17.04 \mu \mathrm{m}$ pure rotational lines from warm molecular gas are seen in both UGC 5101 and Mrk 463e. In UGC 5101 the $\mathrm{S}(3) / \mathrm{S}(1)$ line flux ratio is about 0.5 , while in Mrk $463 \mathrm{e}$ it is $\sim 1.3$, implying warm gas temperatures (assuming LTE) of approximately $300 \mathrm{~K}$ and $400 \mathrm{~K}$, respectively. If the $\mathrm{S}(1)$ line emission is from an unresolved source in both cases, there is $\sim 1.1$ and $0.5 \times 10^{9} \mathrm{M}_{\odot}$ of warm molecular gas in UGC 5101 and Mrk 463e, respectively. These warm molecular gas estimates are $22 \%$ and $48 \%$, respectively, of the total $\mathrm{H}_{2}$ masses (warm plus cold) in these galaxies for $\alpha=0.8 \mathrm{M}_{\odot}\left(\mathrm{K} \mathrm{km} \mathrm{s}^{-1} \mathrm{pc}^{2}\right)^{-1}$ (Solomon et al 1997; Evans et al. 2002).

We detect the 6.2, 7.7, 11.3, and $12.7 \mu \mathrm{m}$ PAH emission features in UGC 5101, and all but the $12.7 \mu \mathrm{m}$ feature in Mrk 1014 (see Figs. 1 \& 2). A weak $7.7 \mu \mathrm{m}$ feature may be present in the SL spectrum of Mrk 463e. In addition, we detect the $16.4 \mu \mathrm{m}$ PAH feature (e.g., Moutou et al. 2000) in UGC 5101. To our knowledge, this is the first detection of this feature in a ULIRG, although it is seen in some nearby starburst galaxies (Sturm et al. 2000; Smith et al. 2004). The $7.7 \mu \mathrm{m}$ line-to-continuum (l/c) ratios we measure for Mrk 1014, Mrk 463e and UGC 5101 are $0.25,0.05$, and 1.1 , respectively. An $\mathrm{l} / \mathrm{c} \geq 1.0$ is usually taken to imply a dominant starburst contribution to the mid-infrared flux (Rigopoulou, et al. 1999). The relatively strong $7.7 \mu \mathrm{m}$, and $11.3 \mu \mathrm{m}$ features in Mrk 1014, may indicate that a circumnuclear starburst is present in this Seyfert 1 galaxy.

We would like to thank Aaron Evans, Bruce Draine, Jacqueline Keane, Bill Reach, J.D. Smith, Jason Surace and Mark Voit for many helpful discussions. We would also like to thank the referee, Eckhard Sturm, for a careful reading of the manuscript. It is also a pleasure to thank the countless people at Cornell University, JPL, Ball Aerospace, Lockheed Martin, and the Spitzer Science Center whose tireless dedication have made success with the IRS and Spitzer a reality. We dedicate these first science results to them. Support for this work was provided by NASA through an award issued by JPL/Caltech.

\section{REFERENCES}

Armus, L., Heckman, T.M, \& Miley, G.K. 1987, AJ, 94, 831.

Blain, A.W., Smail, I., Ivison, R.J., Kneib, J.-P., \& Frayer, D.T. 2002, PhR, 369, 111.

Chiar, J.E., et al. 2000, ApJ, 537, 749

Decin, L., Morris, P.W., Appleton, P.N., Charmandaris, V., \& Armus, L. 2004, ApJ Suppl., in press.

Evans, A.S., Mazzarella, J.M., Surace, J.A., \& Sanders, D.B. 2002, ApJ, 580 .

Genzel, R., Lutz, D., Sturm, E., Egami, E., Kunze, D., et al. 1998, ApJ, 498, 589 .

Goodrich, R.W., Veilleux, S., \& Hill, G.J. 1994, ApJ, 422, 521.

Higdon, S.J.U., et al. 2004, PASP, submitted.

Houck, J.R., et al. 2004, ApJ Suppl., in press.

Huthings, J.B., \& Neff, S.G. 1989, AJ, 97, 1306.

Imanishi, M., Dudley, C.C., \& Maloney, P.R. 2001, ApJ, 558, L93.

Imanishi, M., Terashima, Y., Anabuki, N., \& Nakagawa, T. 2003, ApJ, 596, L167.

Kim, D.C., \& Sanders, D.B. 1998, ApJS, 119, 41.
Lonsdale, C.J., Smith, H.E., \& Lonsdale, C.J. 1995, ApJ, 438, 632.

Lutz, D., Kunze, D., Spoon, H.W.W., \& Thornley, M.D. 1998, A \& A, 333, L75.

Lutz, D., Veilleux, S., \& Genzel, R. 1999, ApJ, 517, L13.

MacKenty, J.W., \& Stockton, A. 1984, ApJ, 283, 64.

Mazzarella, J.M., Gaume, R.A., Soifer, B.T., Graham, J.R., Neugebauer, G., \& Matthews, K. 1991, AJ, 102, 1241.

Mihos, C.J., \& Hernquist, L. 1996, ApJ, 464, 641.

Miller, J.S. \& Goodrich, R.W. 1990, ApJ, 355, 456.

Moshir, et al. 1990, IRAS Faint Source Catalog, V2.0.

Moutou, C., Verstraete, L., Leger, A., Sellgren, K., \& Schmidt, W. 2000, A \& A, 354, L17.

Murphy, T.W. Jr., Armus, L., Matthews, K., Soifer, B.T., Mazzarella, J.M., Shupe, D.L., Strauss, M.A., \& Neugebauer, G. 1996, AJ, 111, 1025.

Reach, W.T., Morris, P., Boulanger, F., \& Okumura, K. 2004 , Icarus, in press. 
Rigopoulou, D., Kunze, D., Lutz, D., Genzel, R., \& Moorwood, A.F.M. 2002, A \& A, 389, 374.

Rigopoulou, Spoon, H.W.W., Genzel, R., Lutz, D., Moorwood, A.F.M., \& Tran, Q.D. 1999, AJ, 118, 2625

Sanders, D.B., et al. 1988a ApJ, 325, 74

Sanders, D.B., Soifer, B.T., Elias, J.H., Neugebauer G., \& Matthews, K. 1988b, ApJ, 328, L35.

Shuder, J.M., \& Osterbrock, D.E. 1981, ApJ, 250, 55.

Smith, J.D.T., et al. 2004, ApJ Suppl., in press.

Soifer, B.T., et al. 1987, ApJ, 320, 238.

Elias, J.H., Lonsdale, C.J., \& Rice, W.L. 1987, ApJ, 320, 238.

Soifer, B.T., et al. 2000, AJ, 119, 509.

Solomon, P.M., Downes, D., Radford, S.J.E., \& Barrett, J.W. 1997, ApJ, 478, 144.

Spoon, H.W.W., Keane, J.V., Tielens, A.G.G.M., Lutz, D., Moorwood, A.F.M., \& Laurent, O. 2002, A \& A, 385, 1022.
Stanford, S.A., Stern, D., van Breugel, W., \& De Breuck, C. 2000, ApJ Suppl., 131, 185.

Strauss, M.A., Huchra, J.P., Davis, M., Yahil, A., Fisher, K.B., \& Tonry, J. 1992, ApJS, 83, 29.

Sturm, E., et al. 2000, A \& A, 358, 481.

Sturm, E., et al. 2002, A \& A, 393, 821.

Tran, Q.D., et al. 2001, ApJ, 552, 527.

Veilleux, S., Kim, D.C., Sanders, D.B., Mazzarella, J.M. \& Soifer, B.T. 1995, ApJ Suppl., 98, 171.

Veilleux, S., et al. 1997, ApJ, 477, 631.

Verma, A., Lutz, D., Sturm, E., Sternberg, A., Genzel, R., \& Vacca, W. 2003, A \& A, 403, 829.

Voit, G.M. 1992, ApJ, 399, 495. 